I.

Aus dem pharmakologischen Institnt zu Bonn.

\title{
Ueber die Wirkung des Brillenschlangengiftes.
}

Von

Dr. med. Fimil Vollmer.

Die Aufmerksamkeit der medicinischen Welt ist in den letzten Jahren wiederbolt den giftigen Kohlenstoffverbindungen zugewandt worden, welche innerhalb lebender Thiere entstehen. So hat man in neuester Zeit das Vorkommen von giftig wirkenden Substanzen in den inneren Organen von Fischen und Mollusken festgestellt. Saotschenkol) giebt an, dass einige Fischgifte das Secret von Hautdrüsen in der Nähe der Riucken- and Schwanzflossen seien. Nach den von Minra und Takesaki ${ }^{2}$ ) im pathologischen Institute zu Tokio veranstalteten Untersuchungen ist bei den Tetrodonen besonders das geschlechtsreife Ovarium stark gifthaltig. Schmidtmann, Salkowsky3), Virchow ${ }^{4}$ ) erklären die Wirkung des Giftes der Miesmuschel (Mytilus edulis), das vorzüglich in der Leber vorhanden ist, der Wirkung von Curare ähnlich. - Auch von den Schlangengiften ist oft angegeben worden, dass ihre Wirkung in vielen Punkten an die von Curare erinnere. Fayrer, Brunton, Wall bestätigen dies für das Gift der Naja tripudians oder Cobra, der gefährlichen Brillenschlange Indiens. Aron leugnet die angegebene Lähmung der motorischen Nervenendigungen, Ragotzi versucht die negativen Resultate Aron's zu erklären. Als mir nun durch die Giite des Herrn Geheimrath Prof. Dr. Binz und indirect durch Herrn Dr. C. R. Francis, jetzt Surgeon General, früher Professor der Medicin des Medicinal College in Calcutta, der auch vor 9 Jahren dem Bonner pharmakologischen Institute einige Glasröhrchen mit getrocknetem

1) Atlas des poissons vénéneux. St. Petersbourg 1887.

2) Zur Localisation des Tetrodongiftes. Virchow's Archiv f. path. Anatomie. Bd. CXXII. 1890.

3) Virchow's Archiv f. path. Anatomie. Bd. CII.

4) Berl. klin. Wochenschr. 1885. Nr. 48. 
Cobragifte geschenkt hatte, solche Giftproben zur Anstellung von Versuchen über geeignete Gegenmittel ${ }^{1}$ ) zur Verfügung gestellt wurden, schien es mir nicht verfehlt, auch über die Wirkung des Cobragiftes besonders auf Nerven- und Muskelsubstanz Versuche zu machen. Die Art der Wirkung des Cobragiftes ist ja trotz vieler Versuche immer noch controvers geblieben. Während die Mehrzahl namentlich der englischen Forscher das Cobragift als ein Nervengift betrachten, ist von deutscher Seite wiederholt versucht worden, das Blut als den ersten Angriffspunkt der Cobravergiftung darzustellen. Die Unwahrscheinlichkeit der letzteren Annahme klarzulegen, war die Hauptaufgabe dieser kleinen Arbeit. Vielleicht bleiben die Resultate nicht ganz unbeachtet und regen zu Arbeiten über die Wirkung des Giftes der Kreuzotter und der Viper auf Nerven- und Muskelsubstanz an, iuber die meines Wissens eingehendere Studien in nenerer Zeit nicht gemacht sind. Was die bekannten Thatsachen über die Natur der Schlangengifte angeht, deren Zurechnung zu den Ptomaïnen eine Vermuthung ist, so verweise ich im Allgemeinen auf die ausgezeichnete Arbeit von A. J. Wall ${ }^{2}$, die aber auch die Frage nach der chemischen genauen Zusammensetzung des Giftes unbeachtet lassen muss. Wie intensiv die Giftkraft auch in den getrockneten Giftproben jahrelang bleibt, bewies mir eine kleine Menge vor $16 \mathrm{Jahren}$ getrockneten Giftes, die an Wirksamkeit einer anderen Probe, dievor $1 \mathrm{Jahregetrocknetwar,} \mathrm{nichtsnachgab.} \mathrm{Ich} \mathrm{stellte}$ mir aus dem getrockneten Gifte 1 proc. Lösungen her, mit denen auch Aron und Ragotzi meist experimentirt haben.

Was die örtlichen Zeichen der Cobravergiftung angeht, so wird mit Uebereinstimmung dem frischen Gifte die Eigenschaft zugeschrieben, an der Bissstelle heftige Entziindung zu erregen. Wall will diese örtliche Reizung sogar als differentialdiagnostisches Merkmal verwerthen zum Unterschiede von minder- oder ungefährlichen Bissen. ${ }^{3}$ ) Dass die Lösungen des getrockneten Giftes dagegen örtiich unwirksam sind, wie einige Autoren behaupten, konnte ich nicht bestätigen. Vielmehr fand ich, wie $\mathrm{W}$ all, bald nach der Einwirkung eines Tropfens Cobralösung von 1 Proe. auf der Conjunctiva von Kaninchen starke Hyperämie, desgleichen in dem subcutanen Gewebe der Injectionsstelle. Auch war der Lymphsack der Frösche, in den sie eine Cobrainjection erhalten hatten, mit einer deutlich gerötheten

1) E. Vollmer, Experimentelle Studien über die Behandlung des Schlangenbisses. Inaug.-Diss. Bonn 1892.

2) Indian snake poisons, their nature and effects. London 1883 .

3) Ibidem. p. 18. 
Flüssigkeit erfüllt, deren Färbung unmöglich von der geringen Verwundung der Haut durch die Pravaz'sche Spritze herrühren konnte. Das allgemeine Vergiftungsbild meiner Versuchsthiere zeigte sie direct nach der Injection unruhig; alsbald aber verfielen sie bis zum Auftreten der objectiven Vergiftungserscheinungen in eine torpide Rube. Die anfangs gesteigerte Athmung ist ohne Zweifel ein Ausdruck der Angst nach dem Injectionsschmerz. I) In einem späteren Vergiftungsstadium fiel mir aber auch eine Steigerung der Athemfrequenz auf, die sich sogar in einer Zunahme der Athemgrösse um fast das Doppelte ibres normalen Werthes in einem Falle kund gab und gasometrisch nachweisen liess.

Die Umrisse des allgemeinen Vergiftungsbildes traten besonders beim Vergleich der Wirkungen gleicher Dosen gleichprocentiger Cobrannd Klapperschlangengiftlösungen ${ }^{2}$ ) auf Versuchsthiere derselben Art and Schwere wie desselben Aiters scharf hervor. Das Hauptzeichen der Vergiftung durch Brillenschlangengift, die schnelle Affection und Lähmung der Athmungscentren, fehlte der Klapperschlangenvergiftung: vollständig. Das zeigen folgende Protokolle:

I. Versuch. a) Ein mittelgrosser Frosch (Weibchen) erhält um $11 \mathrm{~h} .35 \mathrm{~m} .0,5 \mathrm{ccm}$ der 1 proc. Klapperschlangengiftlösung (1884) in den Dorsallymphsack. 11 b. $4 \mathrm{~m}$. Athmung 90. 12 h. keine Riickenlage. $1 \mathrm{~h}$. Athmung unverändert. $2 \mathrm{~h}$. Rückenlage. Um 3 und um $6 \mathrm{~h}$. athmet er noch weiter. Um $8 \mathrm{~h}$. liegt er todt im Wasser. b) Ein gleichgrosser Frosch (Männchen) um dieselbe Zeit mit 0,5 ecm 1 proc. Cobralösung (1875) subeutan vergiftet. $12 \mathrm{~h}$. keine Rückenlage. $12 \mathrm{~h} .15 \mathrm{~m}$. Rückenlage. Athmung $60.12 \mathrm{~h} .20 \mathrm{~m}$. Athmung $40.12 \mathrm{~h} .30 \mathrm{~m}$. Athmung $=0$, todt.

II. Versuch. a) Einem Kaninchen $(700 \mathrm{~g}) 0,5 \mathrm{ccm}$ der 1 proc. Klapperschlangengiftlösung (1884) subcutan in den Riicken. $4 \mathrm{~h} .35 \mathrm{~m}$. Athmung 80. 5 h. $15 \mathrm{~m}$. Athmung 80. Kein Zeichen des Krankseins! $7 \mathrm{~h}, 9 \mathrm{~h}$. unverändert. $7 \mathrm{~h}$. des anderen Morgens liegt es todt im Stalle, ohne Rigor. b) Einem Kaninchen $(820 \mathrm{~g}) 0,5 \mathrm{ccm}$ der Cobralösung (1 Proc., 1875) subcutan injicirt in den Ruieken um dieselbe Zeit. Athmung $80.4 \mathrm{~h} .50 \mathrm{~m}$. Athmung 120. $5 \mathrm{~h}$. Athmung unregelmässig. $5 \mathrm{~h}$. $10 \mathrm{~m}$. Athmung 60. Nicken des Kopfes. $5 \mathrm{~h} .12 \mathrm{~m}$. Athmung 48, Seitenlage. $5 \mathrm{~h} .14 \mathrm{~m}$. Convulsionen, Athmung oberflächlich, stirbt. $7 \mathrm{~h}$. Musculatur durch den starken Rigor mortis bretthart.

1) Aron, Experimentelle Studien über Schlangengift. Zeitschrift für klin. Medicin. Bd. VI. 1883.

2) Herr Prof. Francis hatte auch einige Milligramm getrockneten Klapperschlangengiftes geschickt, die aber leider zu ausgedehnteren Versuchen nicht mehr ausreichten. Ich verbrauchte den Bestand bei den Gegenmittelversuchen fast vollständig. 
Diese Versuche bestätigen also, dass die Stärke des Cobra- nnd Klapperschlangengiftes, wie die Art ibrer Wirksamkeit verschieden ist; besonders der Kaninchenversuch zeigt das: 40 Minuten nach der Einspritzung stirbt das Cobrathier. Die livide Färbung der Ohren and Lippen, die beständige Abnahme der Athemfrequenz und die Zuckungen weisen auf Tod durch Lähmung der Athmung. Wie Wall bemerkt, äbnelt die Vergiftungserscheinung einer aeut gewordenen progressiven Bulbärparalyse. Auch bei der Cobravergiftung werden zuerst die Gebiete des Hypoglossus, Accessorius, Vagus und Facialis afficirt. ${ }^{1}$ ) - Das Klapperschlangenthier überlebt das mit Cobragift behandelte um viele Stunden.

\section{Wie wirkt das Cobragift auf das Blut?}

Ragotzi neigt zu der Ansicht, dass auch bei der Cobravergiftung intravitale Thrombosen eine Rolle spielen, und will dieselben sogar in den Lungen mit der von Filehne angegebenen Methode nachgewiesen haben. Selbst wenn die von Filehne und Silber$\operatorname{man} n^{2}$ ) angestellten Versuche untadelhaft sind, scheint es mir undenkbar, dass durch die minimalen Veränderungen, die Cobradosen, wie Ragotzi ${ }^{3}$ ) sie anwandte, im Blute der Warmblüter hervorrufen, nachweisbare Thrombosirungen entstehen können. Das Blat meiner vergifteten Kaninchen war stets, da ja der Tod durch allmählichen Stillstand der Athmung eintritt, venös. Ausgedehnte Blutgerinnung, die nach Fayrer und Wall bei Einspritzung des frisehen, wie des gelösten Giftes ein häufiger Sectionsbefund sein soll, beobachtete ich nie. Von Veränderungen an den rothen Blutzellen, wie sie Fayrer in dem Blute vergifteter Ratten gesehen hat, habe ich bei Kaninchen nichts finden können. Das Blut der mit einer tödtlichen Gabe von $0,5 \mathrm{ccm} 1$ proc. Cobragiftlösung vergifteten Kaninchen war unter dem Mikroskop normal und von dem gesunder Thiere nicht zu unterscheiden. In der Verdünnung; wie das Gift im Blut circulirte, hatte es den Blutzellen nichts anhaben können. Ich habe auch nie in den inneren Organen, wie Lunge, Leber oder Nieren, meiner vergifteten Kaninchen Hämorrhagien irgend welcher Art vorgefunden. In concentrirter Form schadet das Gift den Blutzellen allerdings auffallend. So konnte ich, wie es auch Ragotzi angiebt, durch Zusatz eines

1) Besonders an Menschen nachgewiesen; vgl. Dr. Hilson, Ind. Med. Gaz. Octoberbeft 1873. p. 254 .

2) Virchow's Archiv f. path. Anatomie. Bd. CXVII 1889.

3) Ueber die Wirkung des Giftes der Naja tripudians. Virchow's Archiv. Bd. CXXII. Heft 2. S. 232. 
Tropfens meiner 1 proc. Giftlösungen $z \mathfrak{u}$ einem Blutpräparate eines Frosches die Gestalt der rothen Blutzellen total verändern. Die elliptischen, biconvexen Zellen verloren ihre scharfen Formen und ibre grînliche Färbung. Sie wurden durchsichtig wie Wasser, und die Kerne traten sehr dentlich hervor. Aehnlich leiden die biconcaven Zellen der Warmbliiter bei directer concentrirter Giftwirkung. Bei einem Präparate meines eigenen Blutes quollen die rothen Blutkörperchen nach Zusatz eines Tropfens der Giftlösung auf, wurden kugelförmig und verloren bald jeden Contour.

Das Klapperschlangengift war gegen die Blutzellen nicht ganz indifferent, wirkte aber lange nicht so scbnell und stark auf sie ein, wie das Cobragift. Diese Einwirkung der concentrirten Giftlösung: auf die Blutzellen hat aber offenbar mit der Affection des gesammten Organismus weniger zu thun, als es den Anschein haben könnte und Heidenschild und Ragotzi meinen. Heidenschild ${ }^{1}$ ) will den Nachweis der veränderten Gerinnungstendenz des Blutes nach Cobraund Klapperschlangenvergiftung geliefert haben. Nach einer Steigerung der Gerinnungsfähigkeit des Blutes stellt sich nach ibm eine Erniedrigung desselben ein. Die Annahme der excessiven Steigerung der ersten Periode erklärt ihm durch Massenthromben den schnellen Cobratod. Aber nun finden sich, wie schon gesagt wurde, Blutgerinnungen keineswegs als Regel bei Sectionen cobravergifteter Thiere. Auch Ragotzi glaubt an intravitale Thrombosen und durch dieselben mögliche secundäre Lähmungen der Nervencentren. Dass die vielleicht vereinzelt möglichen Thrombosen mit dem specifischen Cobratode aber nichts zu thun haben, glaube ich bewiesen zu haben. ${ }^{2}$ ) Das Blut ist vielmehr auch bei der Cobravergiftung, wie etwa bei der Vergiftung durch Strychnin, Atropiv, Morphin, nur Vermittler zwisehen Gift und Nervensystem.

\section{Wie wirkt das Cobragift auf das Herz?}

Das Herz von Fröschen führt, wie alle Beobachter angeben, nach dem Stillstand der Athmung noch längere Zeit deutliche Zusammenziehungen aus, falls das Gift unter die Haut gespritzt wurde. Die Herzganglien sind also wohl für das Cobragift nicht in gleichem Maasse empfänglich, wie die Centren der Medulla oblongata. Nichtsdestoweniger wird auch das Herz von dem Gifte angegriffen. Dasselbe steht bei Fröschen 1 bis 2 Stunden nach der Lähmung der Athmung

1) Ueber die Wirkung des Giftes der Brillenschlange und Klapperschlange. Inaug.-Diss. Dorpat 1886.

2) Vgl. Versuch XVI, XVII, XVIII. 
still, wo es doch sonst noch Tage nach dem Tode weiterschlägt. Bei subcutaner Injection tritt der Herzstillstand um so schneller ein, je grösser die Giftkraft und die Menge der eingespritzten Lösung war. Vollends vermag unmittelbare Einspritzung des Giftes in grössere Venen die Herzthätigkeit mit einem Male zu bemmen.

Ragotzi hat das Froschherz nach subcutaner Anwendung des Giftes stets in Diastole, bei intravenöser Injection hingegen in Systole gesehen. Ich fand es auch bei subcutaner Anwendung in Systole. Wie stark das Cobragift bei directer Einwirkung das Herz angreift, zeigen folgende Protokolle:

III. Versuch. Ein kleiner Frosch wird decapitirt, die Chorda spinalis zerstört und nun das Herz herausgeschnitten und in Wasser gelegt. 30 starke, kräftige Contractionen $10 \mathrm{~h} .20 \mathrm{~m}$. In Cobralösung gehalten (1 Proc., 1875) sind 10 h. $25 \mathrm{~m} .40$ Contractionen, 10 h. $27 \mathrm{~m}$. nur noch $12 \mathrm{zu}$ constatiren, und um $10 \mathrm{~h}, 30 \mathrm{~m}$. steht das Herz in Systole still.

IV. Versuch. Die starke Herzaffection bei intracardialer Injection zeigte sich auch, als ich nach vorgenommener Herzpräparation einer grossen Rana temp. 0,1 ccm Cobralösung (1 Proc., 1875) ins Herz injicirte. Ich wandte eine haarfeine Canüle an und injicirte zur Controle einer gleich grossen Rana dieselbe Menge Wasser in die Ventrikel. 3 Min. nach der Cobrainjection stand das Herz still, während die Wasserinjection (keine physiol. NaCl-Lösung!) die Contractionen nur unmerklich beeinflusste. Nach 11/2 Stunde fand ich noch 40 Contractionen in der Minute. - Bei der intravenösen Einspritzung von $0,5 \mathrm{ccm}$ Cobralösung (1 Proc., 1891) in die Jugularis eines grossen Kaninchens stand das Herz in 1 Minute in Systole still!

Die Versuche Ragotzi's, die Differenz der Befunde Brunton's und Aron's über die Einwirkung des Cobragiftes auf die motorischen Nervenfasern der Peripherie durch die Annahme einer frühzeitigen Herzlähmung und einer dadurch bedingten mangelhaften peripheren Vergiftung bei Aron's Versuchen zu erklären, veranlassten mich, der Herzaction von Fröschen, die grosse Cobradosen subcutan erhalten hatten, besondere Aufmerksamkeit zu schenken. Wie die Protokolle ${ }^{1)}$ beweisen, wird das Herz bei der Verdünung des Giftes, wie dasselbe nach der Resorption in dem Blute und dem Gewebswasser kreist, nur langsam und schwach angegriffen, auch bei zehn- und mehrfach letalen Dosen. Somit fehlt der Vermuthung Ragotzi's die experimentelle Bestätigung. - Wenn Brunton und Fayrer ${ }^{2}$ ) nach der Vergiftung

1) Vgl. Versuch I, IX, X, XI.

2) On the nature and action of the poison of Naja. Proc. Roy. Soc. XXII. Exp. LXXII. 
eine Blutdruckverminderung fanden, die anch Feoctistow ${ }^{1}$ ) nachwies, und zu deren Erklärung er eine Lähmung der Endigungen des Bauchsympathicus annimmt, so sehe ich nach meinen Versuchen von einer Herzparalyse zur Erklärung der auffallenden Blutdrucksenkung, die ich auch nachweisen konnte, ab (vgl. Versuch V). Wenn wir die schnelle Lähmung der motorischen Peripherie sehen, so liegt die Vermuthung auf der Hand, dass auch die Vasomotoren in gleicher Weise geschädigt werden. So erklärt sich die Blutdrucksenkung einfach durch Erweiterung der Blutbahnen infolge einer Vasomotorenparalyse, und wir bedürfen gar nicht der Annahme von ausgedehnten Verlegungen der Gefässe im Lungengebiete einerseits und Pfortadergebiete andererseits, abgesehen davon, dass dieselben bei unseren Versuchen nie nachzuweisen waren. Auch zeigten mir die Blutpräparate von vergifteten Warmblütern keine Formveränderungen, obschon ich Dosen von $1 \mathrm{ccm} 1$ proc. Cobralösung (1875) zur Vergiftung benutzte, die intravitale Thrombosen in Masse hervorrufen konnten. - Die Blutdrucksenkung weisen folgende Versuche aus:

V. Versuch. a) In die Carotis dextra eines circa $1460 \mathrm{~g}$ schweren Kaninchens wird ein Manometer eingeschaltet. Der normale Blutdruck schwankt $4 \mathrm{~h} .30-35 \mathrm{~m}$. zwisehen 30 und $24 \mathrm{~cm}$ der Seala. $4 \mathrm{~h}$. $35 \mathrm{~m}$. Injection von $0,4 \mathrm{ccm}$ der 1 proc. Cobralösung (1891) in die Thoraxhaut. Nach 5 Min. beginnt die Quecksilbersäule zu fallen. Der Druck ist um 4 h. $40 \mathrm{~m}$. dem atmosphärischen gleich. b) Einem gleich schweren Kaninchen injicirte ich um 5 h. 5 m. in die Seite des rechten Oberschenkels die gleiche Menge derselben Lösung. Nach anfänglicher Steigerung von 24 auf 28 sinkt der Druck wieder wie im anderen Versuche.

\section{Wie wirks das Cobragift auf die Muskeln?}

Bei Menschen, die von Cobraschlangen gebissen wurden, hat man Erscheinungen von Seiten der motorischen Apparate beobachtet, die als Coordinationsstörungen gedeutet sind. Aehnliches glaube ich auch bei den Kaninchen und Fröschen gesehen zu haben. In einem bestimmten Stadium der Vergiftung stossen dieselben die hinteren Extremitäten nicht mehr, wie meist normal, gleichzeitig aus, sondern kriechen unbeholfen vorwärts. - Die Reizbarkeit der Muskelsubstanz fand ich beim Frosehe nach directer Einwirkung des Cobragiftes, wie nach Vergiftung durch das Blut herabgesetzt. Nach der subcutanen

1) Ueber die Wirksamkeit des Schlangengiftes auf den thier. Organismus. Mém. de l'académie imp. des sciences de St. Petersbourg. VIl. Série. Tome XXXVI. No. 4. 1888. 
Einspritzung zog diese Affection zwar langsamer, aber stetig heran. Ausser den Protokollen von Versuch VIII, XI, XIII bestätigt dies:

VI. Versuch. Ein grosser Froseh wird an der rechten hinteren Extremität nach Claude-Bernard'scher Methode mit Abbindung der Circulation bei Freilassung des Ischiadicus behandelt. Der freigelegte Nerv wird über ein kleines Deckgläschen gelegt und die lädirte Stelle sorgfältig mit feuchtem Fliesspapier bedeckt. Athmung 100. Darauf $10 \mathrm{~h}$. $25 \mathrm{~m}$. Injection von $0,4 \mathrm{ccm}$ der 1 proc. Cobralösung (1875) in den Dorsallymphsack. $11 \mathrm{~h} .20 \mathrm{~m}$. steht die Athmung. $11 \mathrm{~h} .45 \mathrm{~m}$. Reaction auf mechanische und chemische Reize bleibt aus. Augen werden noch geöffnet und geschlossen. Die Reizbarkeit der Muskel- und Nervensubstanz wird nun mit einem Induetionsstrom gepruft, der durch einen thermoelektrischen Apparat angeregt wird. In der Inductionsrolle sind ein Bïndel Eisenstäbe verschieblich angebracht und mit einer Scala von $0-14$ versehen. $0=$ stärkster, $14=$ schwächster Strom. Die Elektroden werden nun auf Muskelsubstanz und den präp. Ischiadicus gebracht. Die Resultate zeigt nachfolgende Tabelle. Die Zahlen sind die der Seala, bei deren Einstellung die Präparate noch deutlich zuckten. Dies gilt für alle folgenden Tabellen.

\begin{tabular}{|c|c|c|c|c|}
\hline \multirow{2}{*}{ Zeit } & \multicolumn{2}{|c|}{ Vergiftetes Bein } & \multicolumn{2}{|c|}{ Giftfreies Bein } \\
\hline & Gastroenemius & Isohiadicus & Gastrocnemius & Ischiadicus \\
\hline $\begin{array}{rl}12 & \mathrm{~h} \\
1 & \mathrm{~h} \\
2 & \mathrm{~h} \\
3 & \mathrm{~h} \\
4 & \mathrm{~h}\end{array}$ & $\begin{array}{r}10 \\
10 \\
5 \\
0 \\
-\end{array}$ & $\begin{array}{r}10 \\
5 \\
0 \\
- \\
-\end{array}$ & $\begin{array}{r}14 \\
10 \\
10 \\
5 \\
5\end{array}$ & $\begin{array}{l}14 \\
14 \\
14 \\
10 \\
10\end{array}$ \\
\hline
\end{tabular}

Man sieht also, dass die directe Muskelerregbarkeit im vergifteten Bein schneller sinkt, als im giftfreien. Diese Schädigung tritt aber langsamer ein, als die des Ischiadicus. - Fayrer wie Aron berichten, dass durch directe Behandlung mit Cobragift die Reizbarkeit der Muskeln ganz rapid herabgesetzt wird, so zwar, dass selbst starke elektrische Reize sehr bald unwirksam sind. Zum Vergleiche auch der Verschiedenheit des Cobra- und Klapperschlangengiftes stellte ich. folgenden Versuch an:

VII. Versuch. Zwei gleichgrosse Frösche werden decapitirt und die von ihnen gewonnenen Gastrocnemii in Wasser gelegt. Von 3 Uhrschälchen wird eins mit 0,7 proc. Chlornatriumlösung gefüllt. In die beiden anderen wird zu je $0,5 \mathrm{ccm}$ einer 1 proc. Giftlösung ein Zusatz von je $2 \mathrm{cem}$ der 0,7 proc. Chlornatriumlösung gebracht. Um $11 \mathrm{~h} .40 \mathrm{~m}$. wird je ein Gastrocnemins des einen Frosches in ein Giftschälchen gelegt und ein Gastroenemius des zweiten Frosches in die Chlornatriumlösung. Ueber die Befunde der elektrischen Prüfung weist folgende Tabelle aus: 
Ueber die Wirkung des Brillenschlangengiftes.

\begin{tabular}{l|c|c|c}
\hline \hline Zeit & $\begin{array}{c}\text { Gastrocnemius } \\
+0,7 \text { Proc. NaCl }\end{array}$ & $\begin{array}{c}\text { Gastrocnemius } \\
\text { + Cobralösung }\end{array}$ & $\begin{array}{c}\text { Gastrocnemius } \\
\text { + Klapper- } \\
\text { sehlangenlösung }\end{array}$ \\
\hline $11 \mathrm{~h} 40 \mathrm{~m}$ & 14 & 14 & 14 \\
$12 \mathrm{~h}-\mathrm{m}$ & 14 & 10 & 14 \\
$12 \mathrm{~h} 15 \mathrm{~m}$ & 14 & 5 & 14 \\
$12 \mathrm{~h} 30 \mathrm{~m}$ & 14 & 0 & 14 \\
$12 \mathrm{~b} 40 \mathrm{~m}$ & 14 & - & 14
\end{tabular}

Der mit Cobralösung behandelte Gastrocnemius zog sich trotz der ziemlich grossen Verdïnnung des Giftes, je länger er in der Lösung lag, desto mehr zusammen und maass um $12 \mathrm{~h} .40 \mathrm{~m}$. nur noch zwei Drittel sciner früheren Länge. Der in Klapperschlangengiftlösung gelegte büsste weder an Reizbarkeit noch an Form irgendwie ein. Während dieser noch weich war, fiuhlte sich der Cobramuskel um 12 h. $40 \mathrm{~m}$. fest und hart an. Diese starke Schädigung erklärt auch den Stillstand des vergifteten Herzens in Systole, wie die schnell eintretende Todtenstarre. - Ueber die glatte Musculatur fehlen ausreichende Beobachtwagen. Die im Augenblicke des Todes und karz nach demselben lebhafte Peristaltik ist wohl kein Beweis für eine besondere Affection der glatten Darmmuskeln. Dass im Augenblicke des Cobratodes spontan Urin entleert wird, also eine Contractur der Blasenmuskeln eintritt, wie ich mehrfach beobachtete, kommt anch sonst in der Agone vor.

\section{Wie wirlt das Cobragift auf die Nerven?}

Das Nervensystem ist nach Uebereinstimmung der englischen Cobraforscher und Aron's, wie auch Ragotzi's der Ort, von dem das Cobragift in so angenfälliger Weise den Tod auslöst. Dagegen denken Feoctistow, Heidensehild und Ragotri an das Blut als die primäre Stelle der Cobragiftwirkung, besonders bei den Vergiftungen mit dem frischen Virus. - Wie und wo im Nervensystem die Giftwirkung einsetzt, ist anch nach den englischen Autoren noch nicht absolut festgestellt. Fayrer und Brunton nehmen eine Lähmung der motorischen Nervenendigungen als Grund der Lähmung der Glieder, als direct den Tod berbeiführende Wirkung eine sehr sehnelle Lähmung der Athmungsganglien in der Medulla oblongata an. Wall findet auch motorische Paralyse und schreibt dariber: These experiments tend to show that the spinal nervons system is rapidly paralysed by cobrapoison, but that the terminations of the motor nerves only suffer pari passu with the cord itself and the poison has no elective affinity for the ends of the nerves. - Aron, der Wall's Arbeiten noch nicht kannte, giebt die Lähmung der motorischen 
Nervenendigungen nicht $\mathrm{zu}$, findet aber ausgesprochene Rückenmarkslähmung. Ragotzi hat bei Anwendung sehr geringer Giftmengen die motorischen Nervenendigungen gelähmt mit Schonung der Muskelsubstanz und so den Beweis erbracht, dass die Nervensubstanz viel empfindlicher als jene von dem Gifte getroffen wird. - Ich Iasse eine Reihe von Versuchen folgen, die sowohl die noch lange Zeit ziemlich ungeschwächte Herzaction beweisen, als die jedesmal von mir beobachtete Lähmung der motorischen Peripherie des Nervensystems.

VIII. Versuch. Um 10 h. $5 \mathrm{~m}$. einem kleinen Frosche $0,3 \mathrm{ccm}$ 1 proc. Cobralösung (1875) in den Dorsallymphsack. Um $11 \mathrm{~h} .20 \mathrm{~m}$. steht die Athmung. Pulse 34! Nach Präparation des Ischiadicus von diesem keine, vom Muskel mit 6 schwache, mit Rollenabstand 0 deutliche Contractionen.

IX. Versuch. a) Um 11 h. $35 \mathrm{~m}$. Injection von $0,3 \mathrm{ccm} 1$ proc. Cobralösung (1875) einem kleinen Frosche in den Dorsallymphsack. $12 \mathrm{~h}$. $25 \mathrm{~m}$. Rückenlage. $12 \mathrm{~h} .40 \mathrm{~m}$. Herzpräparation. Pulse 44 ! b) $0,3 \mathrm{ccm}$ der 1 proc. Cobralösung (1891) einem kleinen Frosche in den Dorsallymphsack $11 \mathrm{~h} .35 \mathrm{~m}$. Rückenlage schon $12 \mathrm{~h} .15 \mathrm{~m}$. Athmung steht $12 \mathrm{~h} .30 \mathrm{~m}$. Herzpräparation ohne Zeichen der Empfindung. Pulse 50 ! um $12 \mathrm{~h} .35 \mathrm{~m}$.

X. Versuch. Injection von $0,3 \mathrm{cem}$ der 1 proc. Lösung Cobragift (1875) einem Frosche in den Dorsallymphsack um 12 h. $25 \mathrm{~m}$. Rückenlage $12 \mathrm{~h} .55 \mathrm{~m} .12 \mathrm{~h} .58 \mathrm{~m}$. Pulse noch 50 . Injection von $0,2 \mathrm{ccm}$ derselben Lösung ins Herzlumen: Ventrikel stehen um $1 \mathrm{~h}$. in Systole still.

XI. Versuch. a) Eine kleine Rana temp. nach Cl. Bernard präparirt. $11 \mathrm{l}$. Injection von $0,5 \mathrm{cem}$ der 1 proc. Lösung (1875) in den Dorsallymphsack. $11 \mathrm{~h} .30 \mathrm{~m}$. Rückenlage. Athmung steht. $11 \mathrm{~h} .40 \mathrm{~m}$. Blosslegung des Herzens. Pulse 44. Um 12 h. Prüfung der elektrischen Reizbarkeit :

\begin{tabular}{c|c|c|c|c}
\hline \multirow{2}{*}{$\begin{array}{c}\text { Rollen- } \\
\text { abstand }\end{array}$} & \multicolumn{2}{|c|}{ Vergiftetes Bein } & \multicolumn{2}{c}{ Giftfreies Bein } \\
\cline { 2 - 5 } & Muskel & Nerv & Muskel & Nerv \\
\hline 14 & - & - & zuckt & zuckt \\
10 & - & - & $=$ & $=$ \\
6 & zuckt & - & $=$ & $=$ \\
3 & $=$ & - & $=$ & $=$ \\
0 & $=$ & - & $=$ & $=$ \\
& & & &
\end{tabular}

b) Von zwei gleichgrossen Fröschen dem einen $0,1 \mathrm{ccm}$ der 1 proc. Cobralösung von 1891, dem anderen $0,5 \mathrm{ccm}$ in den Dorsallymphsack $2 \mathrm{~h} .25 \mathrm{~m}$. $3 \mathrm{~h} .45 \mathrm{~m}$. steht die Athmung bei beiden. Um $4 \mathrm{~h} .15 \mathrm{~m}$. 
hat der erste noch 60, der zweite noch 42 Pulse. Prüfung der elektrischen Reizbarkeit:

\begin{tabular}{|c|c|c|c|c|}
\hline \multirow{2}{*}{ Zeit } & \multicolumn{2}{|c|}{ Frosch mit $0,1 \mathrm{ccm}$} & \multicolumn{2}{|c|}{ Frosch mit $0,5 \mathrm{ccm}$} \\
\hline & Muskel & Nerv & Muskel & Nerv \\
\hline $4 \mathrm{~h} 30 \mathrm{~m}$ & 14 & $\ldots$ & 14 & - \\
\hline $5 \mathrm{~h} 30 \mathrm{~m}$ & 10 & - & 10 & - \\
\hline $6 \mathrm{~h} 30 \mathrm{~m}$ & 5 & - & 5 & 一 \\
\hline $7 \mathrm{~h} 15 \mathrm{~m}$ & 0 & - & 0 & 一 \\
\hline
\end{tabular}

XII. Versuch. Einer grossen Rana temp. 0,5 cem der 1 proc. Cobralösung von 1875 subcutan in den Dorsallymphsack. Vorher Präparation des Ischiadicus nach Claude Bernard. Injection $10 \mathrm{~h}$. $10 \mathrm{~h} .55 \mathrm{~m}$. Riickenlage. Athmung steht. $11 \mathrm{~h} .10 \mathrm{~m}$. Herzschlag 40 ! Reizung des vergifteten Ischiadicus giebt keine Zuckungen, des giftfreien deutliche.

XIII. Versuch. a) Einer nach Claude Bernard präparirten Rana 0,2 ccm der 1 proc. Oobralösung in den Dorsallymphsack. Athmung $80.11 \mathrm{~h} .40 \mathrm{~m}$. Rüickenlage $12 \mathrm{~h} .25 \mathrm{~m}$. Athmung steht. $13 \mathrm{~b}$. $40 \mathrm{~m}$. Prüfung der elektrischen Reizbarkeit.

\begin{tabular}{c|c|c|c|c}
\hline \multirow{2}{*}{$\begin{array}{c}\text { Rollen- } \\
\text { abstand }\end{array}$} & \multicolumn{2}{|c|}{ Vergiftetes Bein } & \multicolumn{2}{c}{ Giftfreies Bein } \\
\cline { 2 - 5 } & Ischiadicus & Gastrocnemius & Ischiadicus & Gastrocnemius \\
\hline 14 & - & - & zuckt & zuckt \\
10 & - & - & $=$ & $=$ \\
6 & - & zuckt & $=$ & $=$ \\
4 & - & $=$ & $=$ & $=$ \\
0 & - & $=$ & $=$ & $=$
\end{tabular}

b) Einem gleichgrossen Frosche $0,2 \mathrm{~cm}$ der 1 proc. Cobralösung (1875) in den Dorsallymphsack $11 \mathrm{~b}, 40 \mathrm{~m} .12 \mathrm{~h} .20 \mathrm{~m}$. Athmung steht, Rückenlage. Puls 60!

XIV. Versuch. Einem kleinen Frosche um 9 h. $7 \mathrm{~m}$. eine Injection von 0,5 cem Cobralösung (1 Proc., 1875) in den Dorsallymphsack. Athmung $90.9 \mathrm{~h} .55 \mathrm{~m}$. Athmung steht. Präparation beider Ischiadici. Herzaction 40 ! An beiden Ischiadicis sinkt die elektrische Reizbarkeit gleichmässig von Stunde za Stunde und ist um $12 \mathrm{~h}$. an beiden mit dem stärksten zu Gebote stehenden Strome nicht mehr nachzuweisen.

XV. Versuch. Einer kleinen, nach Claude Bernard präparirten Rana temp. 0,5 ccm der 1 proc. Gobralösung (1875) in den Dorsal. lymphsack. Athmung $64.11 \mathrm{~h} .55 \mathrm{~m} .2 \mathrm{~h} .35 \mathrm{~m}$. Nur schwache Reaction bei der Präparation des Ischiadicus des vergifteten Beines. Um $1 \mathrm{~h}$. Vergleich der elektrischen Erregbarkeit von Muskel und Nerven der kranken und gesunden Seite. 


\begin{tabular}{c|c|c|c|c}
\hline \multirow{2}{*}{$\begin{array}{c}\text { Rollen- } \\
\text { abstand }\end{array}$} & \multicolumn{2}{|c|}{ Vergiftetes Bein } & \multicolumn{2}{c}{ Giftfreies Bein } \\
\cline { 2 - 5 } & Muskel & Nerv & Muskel & Nerv \\
\hline \hline 12 & - & - & zuckt & zuckt \\
10 & - & - & $=$ & $=$ \\
8 & - & - & $=$ & $=$ \\
6 & zuckt & - & $=$ & $=$ \\
4 & $=$ & - & $=$ & $=$ \\
2 & $=$ & - & $=$ & $=$ \\
0 & $=$ & - & $=$ & $=$
\end{tabular}

Bei Rollenabstand 2 und 0 wurden vom kranken Nerven aus lebhafte Mitbewegungen des abgebundenen Beines beobachtet!

Nach diesen Versuchen steht die Lähmung der motorischen Nervenendigungen wohl endgültig fest. Von einer ausgesprochenen schnelleren Lähmung des Rückgrates sah ich nichts. Hingegen fand ich noch Erhaltung der Reflexe, solange als das Nervensystem überhaupt noch leistungsfähig geblieben war, und es gelang mir, die unteren Extremitäten eines Frosches so lange vom Halsmarke aus zu Zuckungen zu bringen, wie vom präparirten Ischiadicus a aus. Wie Wall glaube auch ich sagen zu dürfen, dass die motorischen Nervenendigungen gelähmt werden, dass diese Lähmung aber gleichen Schritt hält mit der Rückenmarksparalyse. Was die curareähnliche Vorliebe des Cobragiftes für die motorischen Nervenendigungen angeht, für die Fayrer und Brunton eintreten, so genügt zur Erklärung aller Vergiftungserscheinungen die Annahme, dass das Nervensystem im Ganzen intensiver und schneller durch das Gift geschädigt wird, als das Muskelsystem.

Die Einwirkung des Cobragiftes auf das centrale Nervensystem ist durch die bisherigen Experimente ausser Frage gestellt. Auch die von mir beobachtete Steigerung der Athemgrösse nach Einspritzung des Cobragiftes darf wobl als ein Beweis dieser centralen Affection, besonders der Medulla oblongata angesehen werden. Wie wirkt nun aber das Cobragift auf das Athemcentrum? Ist die centrale Lähmung veranlasst durch allgemeine vom Gift im Blate hervorgerufene Veränderungen, die Form und Funetion der rothen Blutzellen zerstören? Finden Thromben ibren Weg in das Athmungscentrum? Oder ist die Giftwirkung eine directe auf Ganglien und Nervenfasern selbst? Diese Fragen zu entscheiden, veranstaltete ich die folgenden Versuche: Bekanntlich vertragen Frösche sebr gut eine Ersetzang des Blutes durch 0,7 proc. Chlornatriumlösung. Sie leben nach dieser totalen Transfusion noch viele Stunden auch obne rothe Blutzellen, und Athemund Herzbewegungen bleiben lange nach der natürlich vorsichtig und 
geschickt auszuführenden Operation normal. Wird nun einem solchen blutlosen Frosche, bei dem also von Gerinnung und Thrombose keine Rede sein kann, Cobragift unter die Haut gespritzt, und treten dann doch die gewöhnlichen Vergiftnngserscheinungen auf, so darf der Beweis erbracht sein, dass Cobragift die Nervenganglien and -fasern primär schädigt.

XVI. Versuch. Finem starken grossen Frosche, Männchen, wird die rechte Cruralvene und die linke Cruralarterie freigelegt. Erstere wird peripher nnterbunden und in den centralen Theil eine haarfeine Canüle eingeführt, die durch einen Gummischlauch mit einer Burette in Verbindung steht. Ueber die Canüle und Venenwand wird wieder eine Ligatur gelegt. Darauf wird die Cruralarterie des linken Beines geöffnet und der Klemmhaken des Gummischlauches geöffnet. Die physiologische Kochsalzlösung sinkt in der Burette, mischt sich mit dem Blute immer mehr und spült dasselbe zuletzt ganz aus dem Gefässsystem, so dass bald aus der Arterie die wasserhelle Salzlösung strömt. Nach halbstündiger Durchspülung sind um $6 \mathrm{~h} .37 \mathrm{~m} .24 \mathrm{ccm}$ der Salzlösung durch das Blutgefässsystem gelaufen. Die Cruralarterie wird wie die Cruralvene nach Herausnahme der Canüle central ligirt und der Frosch ins Wasser gesetzt. Erholt sich bald. Athmung 60. Reflexe prompt. Rückenlage sofort verlassen. Zur Controle wird einem gleichgrossen zweiten Frosche zur gleichen Zeit die gleiche Dosis Cobragift eingespritzt.

a) $6 \mathrm{~h} .45 \mathrm{~m}$. Dem Salzfrosche $0,4 \mathrm{cem}$ der 1 proc. Cobralösung von 1891 in den Dorsallymphsack. $6 \mathrm{~h} .50 \mathrm{~m}$. Athmung 60. $7 \mathrm{~h} .15 \mathrm{~m}$. Riickenlage, auf chemische Reize wieder verlassen. $7 \mathrm{~h} .20 \mathrm{~m}$. Athmung steht. 7 h. $25 \mathrm{~m}$. Pulse 44 . Lungen schneeweiss. Herz wachsgelb, desgl. die Musculatur. Der Typus der Cobravergiftung mit Wanken des Kopfes, Ataxie der Extremitäten und den eigenthümlichen ziehenden Bewegungen der Hals- und Bauchmusculatur, wie bei den normalen Fröschen! b) $6 \mathrm{~h}$. $45 \mathrm{~m}$. Dem gleichgrossen Frosche $0,4 \mathrm{ccm}$ der Cobralösung (1 Proc., 1891 ) in den Dorsallymphsack. 7 h. Athmung unregelmässig. 7 h. $20 \mathrm{~m}$. Athmung steht. Riuckenlage. 7 h. 25 m. Pulse 46 !

XVII. Versuch. a) Einem grossen, starken Frosche (Weibchen) innerhalb einer halben Stunde $40 \mathrm{ccm}$ der physiologisehen Kochsalzlösung durch das Gefässsystem. Dann ins Wasser. $5 \mathrm{~h} .35 \mathrm{~m}$. Athmung tief, aber langsam, 22. 5 h. 40 m. Athmung 30.5 h. 50 m. Athmung 50 . Injection von $0,2 \mathrm{ccm}$ der Cobralösung ( 1 Proc., 1891) in den Dorsallymphsack. 6 h. 15 m. Athmung steht. Pulse 48 . b) Ein gleichgrosser Frosch um dieselbe Zeit mit derselben Dosis vergiftet. $6 \mathrm{~h}$. Athmung 50. $6 \mathrm{~h}$. $15 \mathrm{~m}$. Athmung 20 . $6 \mathrm{~h} .20 \mathrm{~m}$. Riickenlage. Athmung 10. $6 \mathrm{~h} .25 \mathrm{~m}$. Athmung steht. Herzschläge 80 !

Die Resultate der Versuche im Ganzen sind also kurz folgende:

1) Eine 16 jährige Aufbewahrungsdauer des getrockneten Cobragiftes hat die Intensität und Art der Giftwirkung nicht beeinträchtigt. 
14 I. VoLLmer, Ueber die Wirkung des Brillenschlangengiftes.

2) Auch nach eingetretener Lähmung der Atbmung schlug das Herz der mit $0,3-0,5 \mathrm{ccm} 1$ proc. Cobragiftlösung vergifteten Frösche noch so schnell und stark, dass die Circulation nicht geschädigt sein konnte.

3) Die Lähmung der motorischen Peripherie des Nervensystems war deutlich.

4) Mechanische Kreislaufstörungen und wesentliche Blutschädigungen sind zur Erklärung der schnellen Affection des Athemcentrums auszuschliessen. 